\title{
ON THE NUMBER OF REPRESENTATIONS OF AN INTEGER AS A SUM OR DIFFERENCE OF TWO CUBES*
}

BY E. T. BELL

1. Introduction and Summary. Let $C(n)$ denote the number of integer solutions $(x, y)=(\xi, \eta), \xi>0, \eta>0$, of $x^{3}+y^{3}=n$, the pair $(\xi, \eta),(\eta, \xi)$ being considered as a single solution, and $D(n)$ the number of integer solutions $(x, y), x>0, y>0$ of $x^{3}-y^{3}=n, n>0$. If the pair $(\xi, \eta),(\eta, \xi)$ in $C(n)$ be counted as two solutions, the total number is evidently $2 C(n)$ or $2 C(n)-1$ according as $n$ is not or is the double of an integer cube $>0$. No determination of $C(n), D(n)$ seems to have been made. It will be of interest therefore to record forms of these functions depending only upon the real divisors of $n$, in analogy to the classical results for $x^{2} \pm y^{2}=n$. These forms also indicate fairly expeditious means for finding all the resolutions of $n$ into a sum or difference of two cubes.

We denote by $\psi(z)$ the well known function whose value is 1 or 0 according as $z$ is or is not an integer square $\geq 0$. In the sequel only integer arguments $z$ occur. For $S(n) \equiv C(n)$ or $D(n)$ we find the following:

$$
\begin{gathered}
n \equiv 0 \bmod 3, \quad n \equiv 0 \bmod 9, \quad S(n)=0 . \\
n \equiv 0 \bmod 9, \quad S(n)=\sum \psi\left(4 t-3 d^{2}\right),
\end{gathered}
$$

the $\sum$ extending to all pairs $(t, d)$ of conjugate divisors of $n / 9$ such that

$$
\frac{1}{3} \sqrt[3]{n}<d \leqq \frac{1}{3} \sqrt[3]{4 n}, \text { or } d<\frac{1}{3} \sqrt[3]{n}
$$

according as $S=C$ or $D$.

(3) $\quad n \equiv \pm 1 \bmod 3, \quad S(n)=\sum \psi\left(\frac{4 t-3 d^{2}}{3}\right)$,

* Presented to the Society, San Francisco Section, June 19, 1925. 
the $\sum$ extending to all pairs $(t, d)$ of conjugate divisors of $n$ such that $d \equiv \pm 1 \bmod 3$, and

$$
\sqrt[3]{n}<d \leqq \sqrt[3]{4 n}, \text { or } d<\sqrt[3]{n}
$$

according as $S=C, D$, the upper or the lower sign \pm being taken throughout.

Corresponding to the exact formulas of Gauss and Eisenstein in the case of two squares for the number of lattice points in the circle of radius $\sqrt{n}$ with center at the origin, we have here

$$
\sum_{r=1}^{n} C(r)=\frac{1}{2}\left\{[\sqrt[3]{n / 2}]+\sum_{r=1}^{N}\left[\sqrt[3]{n-r^{3}}\right]\right\},
$$

where $N=[\sqrt[3]{n}]$, and $[z]=$ the greatest integer $\leqq z$. This and the formulas for the circle are obvious cases of the following. Let $M(n)$ denote the total number of pairs of integers $(x, y), x>0, y>0$, satisfying $n=a x^{r}+b y^{s}$, where $a, b, r, s$ are integers $>0$. Then

$$
\sum_{\alpha=1}^{n} M(\alpha)=\sum_{\alpha=1}^{N}\left[\sqrt[v]{\left(n-b \alpha^{s}\right) / a}\right], N \equiv[\sqrt[s]{n / b}] .
$$

2. Proofs of $(1)-(5)$. Putting

$$
n=d t=(x+y)\left(x^{2}-x y+y^{2}\right)=x^{3}+y^{3},
$$

we take $x+y=d, \quad x^{2}-x y+y^{2}=t$, and hence

$$
3 x^{2}-3 d x+\left(d^{2}-t\right)=0 .
$$

The necessary and sufficient condition that this equation have an integer root is $4 t-d^{2}=3 w^{2}, w$ an integer. Since $4 t-d^{2}$ and $d$ are of like parity, so also are $d, w$. Provided $d>w$, the values of $(x, y)$ are then $\frac{1}{2}(d \pm w)$ in either order, and hence from the definition of $C(n)$ we may take $w \geqq 0, x=\frac{1}{2}(d+w), \quad y=\frac{1}{2}(d-w)$. From $w^{2} \geqq 0$, $d>w$, we have at once the first inequality stated in (3).

For a particular $d$ there is one and only one $t$, and hence not more than one $w$; so that each $d$ gives not more than one $(x, y)$, and the $(x, y)$ 's for two different $d$ 's are distinct. 
The total number of pairs $(x, y)$ is therefore equal to the number of integer squares $\left(4 t-d^{2}\right) / 3$. Of the 9 possible cases only the following give $4 t-d^{2} \equiv 0 \bmod 3$,

$$
(n, d, t) \equiv(0,0,0), \quad(1,1,1), \quad(2,2,1) \bmod 3 .
$$

In the case $(0,0,0)$ we set $(d, t)=\left(3 d_{1}, 3 t_{1}\right)$, and hence $n=9 n_{1}\left(n_{1}=d_{1} t_{1}\right)$. Reduction of the conditions upon $d$, and final replacement of $\left(n_{1}, d_{1}\right)$ by $(n, d)$ with the proper qualifications gives $C(n)$ as stated in (2); the $C(n)$ of (1), (3) are equivalent to what precedes.

For $D(n)$ we proceed similarly from

$$
n=d t=(x-y)\left(x^{2}+x y+y^{2}\right)=x^{3}-y^{3},
$$

setting $x-y=d$, and hence $3 x^{2}-3 d x+\left(d^{2}-t\right)=0$. The condition for an integer root is as before, and it is seen immediately that we can take $x=\frac{1}{2}(w+d)$, $y=\frac{1}{2}(w-d), w>0$, with the condition $w-d>0$, which includes necessarily $w>0$. Hence the sole condition is $d<\sqrt[3]{n}$.

The formula (5) is either evident or it is the immediate consequence of equating coefficients of $q^{n}$ in the expansions of

where

$$
\frac{1}{1-q} x\left\{f\left(q^{a}\right) g\left(q^{b}\right)\right\}, \quad\left\{\frac{f\left(q^{a}\right)}{1-q}\right\} x g\left(q^{b}\right),
$$

$$
f(q)=\sum_{0}^{\infty} q^{n^{r}}, \quad g(q)=\sum_{0}^{\infty} q^{n^{s}}, \quad|q|<1 .
$$

3. Decomposition into Cubes. The least favorable situation is that in which $n$ is so large that its resolution into factors is impracticable. It will be sufficient to indicate the process for $C(n)$ when $n \equiv 0 \bmod 9$. We write down the $[\sqrt[3]{4 n} / 3]-[\sqrt[3]{n} / 3]$ integers satisfying the first inequality in (2), test which of these divide $n / 9$, obtaining thus the pairs $\left(d_{i}, t_{i}\right)(i=1,2, \ldots)$ of conjugate divisors. If of these $i=a, b, \cdots$ give all pairs such that $4 t_{j}-3 d_{j}^{2}$ $(j=a, b, \ldots)$ is a square, all the solutions of $x^{3}+y^{3}=n$ with $x>0, y>0$ are 
$x=\frac{1}{2}\left\{3 d_{j}+\sqrt{4 t_{j}-3 d_{j}^{2}}\right\}, \quad y=\frac{1}{2}\left\{3 d_{j}-\sqrt{4 t_{j}-3 d_{j}^{2}}\right\}$.

Numerous devices for shortening the computations are suggested by numerical work, whether or not the prime factor resolution of $n$ be feasible.

As an immediate consequence of (2) we note that 9 is the only prime multiple of 9 which is the sum of two cubes $>0$; from (3) the only solution $x>0, y>0$ of $x^{3}+y^{3}=p^{2}, p$ prime, is $(x, y, p)=(1,2,3)$, etc. It is not difficult to obtain from (1) - (3) the known types of impossible equations $x^{3} \pm y^{3}=n$, except when $n$ is a cube, and some others that do not seem to have been stated.

The University of Washington

\section{CONTACT CURVES OF THE RATIONAL PLANE CUBIC*}

BY L. W. GRIFFITHS

1. Introduction. Contact conics and hyperosculating curves of the rational cubic have been discussed by Winger. $t$ Likewise some account has been given of curves of order $n$ which cut the cubic, rational or elliptic, in $(3 n-1)$ coincident points. $\$$ There remains the question of contact curves of order $n(n>2)$ whose contacts are of lower orders. This paper considers that question for the rational cubic, with results which hold for $n \geqq 1$ and for contacts of any order.

If the cubic is taken in the canonical form

$$
x_{1}=3 t^{2}, \quad x_{2}=3 t, \quad x_{3}=t^{3}+1,
$$

a necessary and sufficient condition that a set of $3 n$

* Presented to the Society, San Francisco Section, December 22, 1923.

$\dagger$ Involutions on the rational cubic, this Bulletin, vol.25 (1918), p. 27.

$\ddagger$ Winger, Some generalizations of the satellite theory, this Bulletin, vol. 26 (1919), p. 75. 